\title{
The use of horticultural therapy for older adults with dementia
}

To achieve psychosocial and physical objectives to improve general well-being through nature/plant activities.

Ellen Dehennin - occupational therapist at University Psychiatric Center KU Leuven, Kortenberg, Belgium

\section{Horticultural therapy \\ " A process that uses plant-related activities through which participants strive to improve their well-being through active and passive involvement" (13)}

Horticultural therapy can be used as a non-pharmacological approach in the care for people with dementia.

It can be an important part of person-centered care and positive person work, which involves engagement of the person with dementia in meaningful activities.

Therefore is it important to know a person's life history.

Sense of self can be reached by caring for specific psychological needs of people with dementia: identity, occupation and inclusion.

These feelings of success can be achieved by collaborating with others during horticultural activities, spending a relaxing moment in a suitable environment or experiencing the joy of creating things. $(5,10,11)$

Horticultural therapy is a multidisciplinary matter.

As a living medium, it requires planning and implementation.
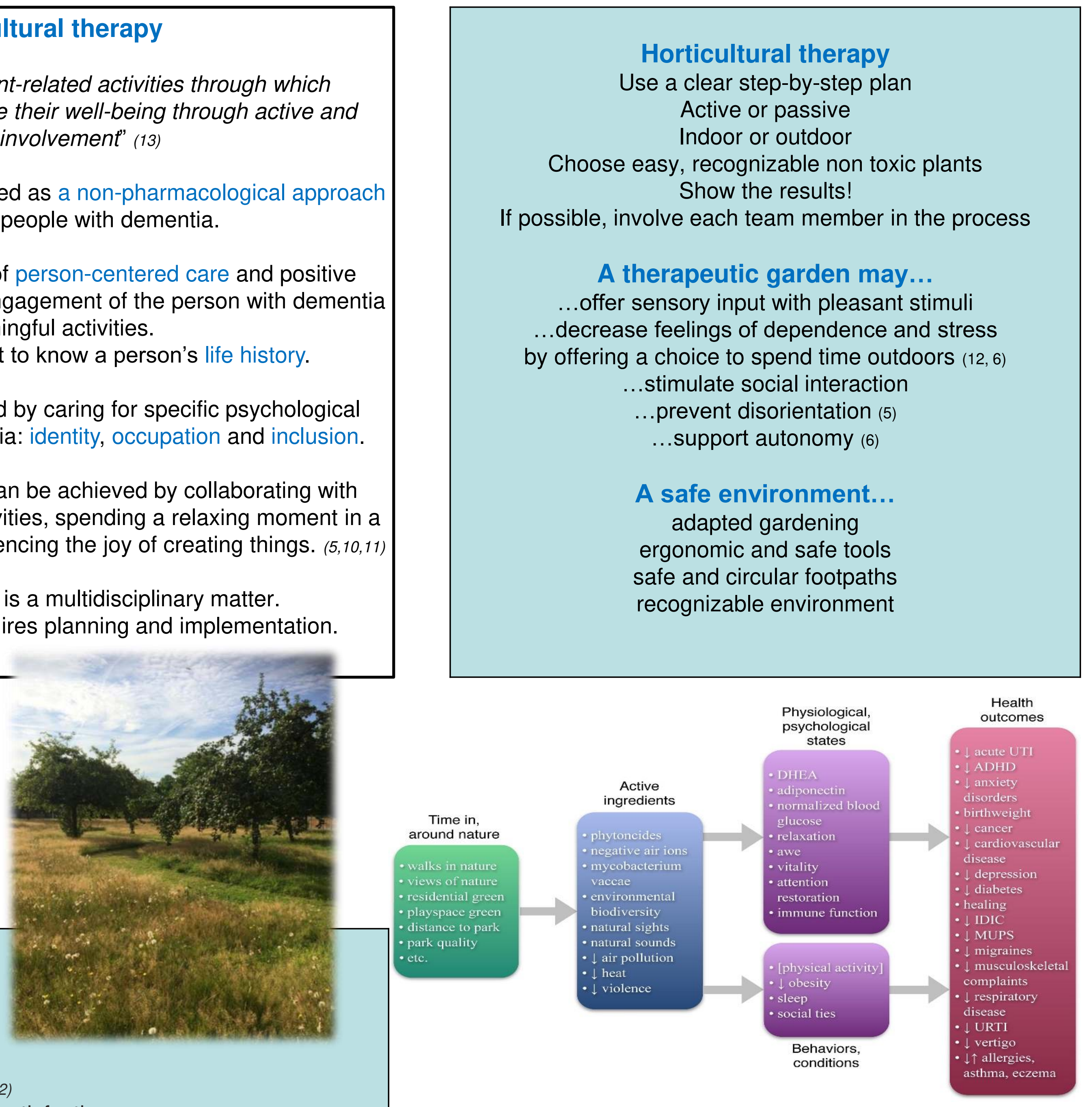

Physical objectives

Increased physical activity (1)

Better sleep quality and sleep/wake rhythm $(1,9)$

Improvement of immune system, cardiac functions, diabetes and obesity, lowering blood pressure, figure above (3)

Faster recovery from illness (9)

Fall prevention and higher absorption of vitamin $\mathrm{D}(2,9)$ Reduction of the need for anxiolytic, sedative or antipsychotic medication (5)

Reduction of pain perception (2)

1. Lee Y. et al, 2008. Effects of indoor gardening on sleep, agitation, and cognition in dementia patients - a pilot study. Intern, journal of geriatric psychiatry $23: 485-489$

2. Detweiler M. et al., 2012, What is the evidence to support the use of therapeutic gardens for the elderly?, Psychiatry investing. 9 (2): $100-110$.

3. Ming Kuo, 2015, How might contact with nature promote human health? Promising mechanisms and a possible central pathway, front. Psychol., 25 aug. 2015

4. M.M. Tse, Therapeutic effects of an indoor gardening programme for older people living in nursing homes, J Clin Nurs, Vol. 19, 2010,949 -95

5. Blake M., Gary, M., 2016, Horticultural therapy in dementia care: a literature review, Nursing Standard, Vol. 30, Iss.21: 41

6. Hewitt P. et al, 2013, Does a structured gardening programme improve well-being in young-onset dementia, British Journal of occupational therapy, $76,8,355-361$

7. Olsson et al., 2013, Persons with early-stage dementia reflect on being outdoors, Aging \& Mental health. 17, 7, 793-800

8. Edwards CA. et al, 2013, An evaluation of a therapeutic garden's influence on the quality of life of aged residents with dementia, Dementia, $12,4,494-510$.

9. Fisher center for Alzheimer's Research Foundation, therapeutic activities

10. Mitchell G., Agnelli J., 2015, non-pharmacological approaches for alleviating distress in dementia care, nursing standard, 30,13, 38-44

11. Mitchell G., Agnelli J., 2015, Person-centered care for people living with dementia: Kitwood reconsidered, Nursing standard, $30,7,46-50$

12. Scherder EJ, et al., 2010, the more physical inactivity, the more agitation in dementia, international psychogeriatrics, 22, 8, 1204-1208.

13. Gonzalez M.T., et al, 2009, Therapeutic horticulture in clinical depression: a prospective study, Res Theory Nurs Pract, 23, 312-328. 